\title{
Sistema automático de reconocimiento de frutas basado en visión por computador
}

\author{
Automatic recognition system of fruits based computer vision
}

\author{
Christian Montoya Holguín ${ }^{1} \quad$ Jimmy Alexander Cortés Osorio ${ }^{2} \quad$ José Andrés Chaves Osorio $^{2}$
}

Recibido 20 de junio de 2013, aceptado 5 de mayo de 2014

Received: June 20, 2013 Accepted: May 5, 2014

\begin{abstract}
RESUMEN
Este trabajo presenta un sistema de reconocimiento capaz de identificar una fruta tropical latinoamericana de entre un conjunto, establecido en una base de datos, utilizando técnicas de visión por computador. La investigación realizada permitió comparar los clasificadores KNN y bayesiano y los modelos del color RGB y HSV, junto con las características de tamaño y forma usadas previamente por investigadores de esta área en países como Malasia, Brasil y Estados Unidos. Para la clase de frutas definidas en esta investigación se determinó que las características que mejor las describieron fueron los valores medios de los canales RGB y la longitud de los ejes mayor y menor cuando se usaba el clasificador bayesiano, proceso que permitió obtener resultados con una exactitud igual al 90\% en las pruebas realizadas, encontrándose que no siempre el seleccionar una mayor cantidad de variables para formar el vector descriptor permite que los clasificadores entreguen una respuesta más acertada, en este sentido es importante considerar que entre las variables de estudio debe presentarse un valor bajo de dependencia o correlación. La síntesis del desarrollo del proyecto dio como resultado la construcción de una báscula electrónica capaz de clasificar frutas, dispositivo que pretende contribuir a la solución del problema de identificación y clasificación de productos agrícolas en los supermercados.
\end{abstract}

Palabras clave: Clasificador, computador, frutas, imágenes, patrones, procesamiento, reconocimiento, visión.

\begin{abstract}
This paper presents a computer recognition system which is able to identify a Latin American tropical fruit, from a set previously defined in a database, using computer vision techniques. This study allows for comparison of the KNN and Bayesian classifiers and the RGB and HSV color models, along with the size and shape characteristics previously used by researchers in this area, in countries such as: Malaysia, Brazil and the United States. For the class of fruits defined in this research, the characteristics that best described them were the mean values of the RGB channels and the length of the major and minor axes, when the Bayesian classifier was used, a process that yielded results with an accuracy equal to $90 \%$ in the tests carried out. It was found that the selection of a larger number of variables, to form the classifier descriptor vector, does not always allow for the delivery of a more accurate response. In this sense, it is important to consider that there should be a low value of dependence or correlation among the variables studied.

The project also resulted in the development of a calibrated video electronic scale prototype, capable of sorting fruit, device that aims to contribute to the solution of the problem of identification and classification of agricultural products in supermarkets.
\end{abstract}

Keywords: Classifier, computer, fruit, images, patterns, processing, recognition, vision.

1 Facultad de Ingenierías. Universidad Tecnológica de Pereira. Pereira, Colombia. E-mail: moncrish@utp.edu.co

2 Facultad de Ciencias Básicas. Universidad Tecnológica de Pereira. Pereira, Colombia.

E-mail: jacoper@utp.edu.co; jachaves@utp.edu.co 


\section{INTRODUCCIÓN}

El proceso de etiquetamiento de productos en los supermercados para su posterior facturación y cobro ha evolucionado desde que se generaba una etiqueta de papel para marcar cada producto con su precio específico, pasando por el código de barras hasta llegar ahora a la tecnología RFID [1] (Radio Frequency Identification).

Esta evolución se ha dado con el fin de disminuir errores en el cobro por causa de la digitación del precio por parte del cajero, como también en la intención de mejorar el grado de satisfacción del usuario por agilizar las colas en los puntos de pago.

No obstante, el proceso de facturación de frutas y verduras vendidas al detalle sigue llevándose a cabo por personal encargado de identificar la fruta o verdura, digitar el código correspondiente y obtener así su valor y posterior factura [2]; debido a que, por ser productos naturales, frescos y que los consumidores desean elegir a su propio gusto y en distintas cantidades, no llevan códigos de barras ni etiquetas RFID. En el contexto de los supermercados, la tecnología RFID permite el acceso remoto desde dispositivos lectores a etiquetas RFID que se adhieren a los productos que se desean identificar almacenando dentro de ellas un código que representa al producto en una base de datos o su precio característico, como lo hace el código de barras, pero que a diferencia de este no requiere un contacto directo con el producto, pues la forma de acceder a la información se logra de manera remota [3]. De esta manera se brinda un mayor grado de automatización en el proceso de facturación de productos en caja, pues al tener, a modo de ejemplo, cierto número de productos (identificados con etiquetas RFID) seleccionados por el usuario dentro de un carro de supermercado y llevar este hasta cierta línea imaginaria donde se encuentra el lector remoto, la factura se generaría en cuestión de segundos y contendría cada uno de los productos seleccionados por el usuario. Pero como se explicó anteriormente, la sección del supermercado donde se encuentran las frutas vendidas al detalle presenta un problema para su automatización o modernización debido a que su funcionamiento es distinto, pues allí el precio de cada artículo está relacionado con su masa y teniendo en cuenta que para una misma clase de fruta cada una en particular presenta un peso diferente, sería necesario identificar cada una de ellas con un código distinto, así pertenezcan a una misma clase, convirtiéndose en una tarea ardua al tener que etiquetar cada unidad con un código de barras; y al ser productos naturales sería conveniente que no contuvieran elementos electrónicos como las tarjetas RFID. Eso hace que para alcanzar gran parte de automatización de un supermercado se deban presentar soluciones alternativas en la sección de frutas y verduras. De esta manera, con el fin de contribuir a la modernización de los supermercados se propone en este trabajo el desarrollo de un sistema basado en visión por computador que esté en capacidad de realizar la percepción autónoma e identificación de la frutas comúnmente encontradas en un supermercado, reconociendo que la inclusión de sistemas electrónicos e informáticos en sistemas de producción ha contribuido con múltiples beneficios, como son la reducción de tiempos, costos y minimización de errores en la ejecución de tareas, logrando así una mayor optimización en el proceso de selección, así como un incremento significativo de la productividad.

Por lo tanto, en la consecución del objetivo del sistema de visión el documento ilustrará las etapas de segmentación, descripción, reconocimiento e interpretación, utilizando para las últimas los algoritmos de clasificación KNN y bayesiano, ambos considerados en la literatura estudiada como algoritmos apropiados y eficaces para este tipo de reconocimiento [4-6]. Todo esto es validado sobre una muestra de la población total de frutas comúnmente encontradas en un supermercado latinoamericano.

La muestra indicada se encuentra constituida por las siguientes frutas: manzana verde, manzana roja, mango Tommy, naranja Tangelo, maracuyá, granadilla, kiwi, guayaba y banano (ver Figura 1). Emprender un proceso investigativo que tiene como objeto de estudio a las frutas es de gran relevancia en un país tropical como Colombia, donde se cultiva una enorme variedad de las mismas, siendo en muchos casos productos de exportación. Las labores investigativas cobran relevancia cuando se pueden orientar, por ejemplo, a la creación de nuevas herramientas y/o estrategias que contribuyan al mejoramiento de los procesos productivos aplicados al sector agrícola, con el fin de aumentar los niveles de calidad y así cumplir con los estándares del mercado. 

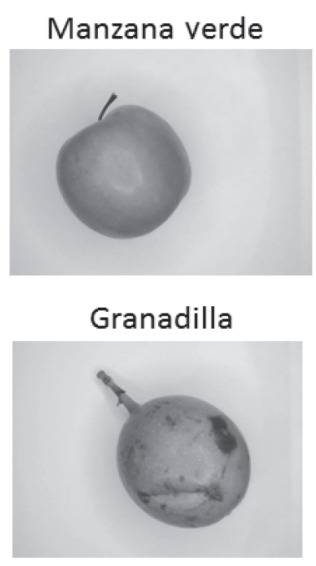

Mango Tommy

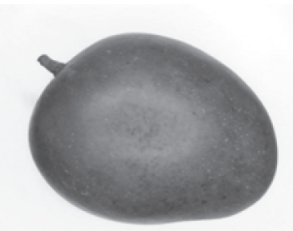

Guayaba

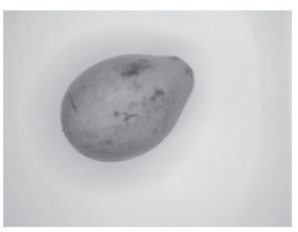

Maracuyá

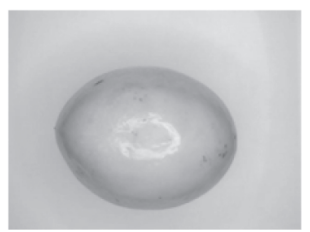

Figura 1. Muestra de frutas del clasificador desarrollado.

\section{ANÁLISIS TEÓRICO-PRÁCTICO}

\section{Revisión de la literatura}

Es interesante observar cómo el problema de clasificación de frutas ha sido de particular interés para los investigadores en el mundo, pues en la revisión de la literatura existente se encontró una gran variedad de artículos relacionados con el procesamiento y clasificación de frutas mediante visión por computador.

A continuación se presentarán los trabajos que tuvieron mayor correspondencia con el problema tratado en esta investigación, empezando con el artículo [7], el cual (observando su fecha de publicación) deja entrever que los desarrollos alrededor de este tema se han venido realizando desde hace varias décadas.

En [7] se plantea el diseño de un sistema de clasificación de frutas de alta velocidad basado solamente en la característica de color. Para este objetivo se utiliza el espacio de color HSI y se concluye que el modo más rápido para alcanzar la clasificación es utilizando solamente la componente $\mathrm{H}$ del modelo y se especifica que para mejorar la precisión y probabilidad de éxito en la respuesta se debe trabajar con los componentes de saturación (S) e intensidad (I), estos presentan cierta desventaja al depender de las condiciones de iluminación, precisando entonces un alumbrado uniforme en las frutas con el objetivo de mantener las condiciones de iluminación constantes.

Dentro de trabajos desarrollados más puntualmente para supermercados se presenta el trabajo de R.M Bolle, J.H Connell, N. Haas, R. Mohan y G.Taubin conocido como "Veggie Vision" (1996) [5] del Centro de investigación TJ Watson de IBM; el fin de este trabajo era facilitar el reconocimiento de frutas y verduras en la caja registradora.

Para esto integraron el sistema de visión a una báscula, como se ilustra en la Figura 2, dentro de un recinto opaco, donde la cámara se encuentra mirando hacia arriba debajo de un plato transparente donde son colocadas las frutas y verduras; para contrarrestar las variaciones de luz en el supermercado, iluminan el producto con luz fluorescente e incorporan dos filtros de polarización, uno lineal que cubre las fuentes de polarización internas y un segundo filtro de polarización sobre la cámara, ortogonal al primero; la idea es evitar la reflexión especular ${ }^{3}$ obteniendo así buenas imágenes a color de objetos brillantes.

Para lograr una buena clasificación, el sistema describe o toma del producto las siguientes características: color, textura, tamaño, forma y densidad de área (masa/área). Para segmentar el producto en la

3 En óptica se llama reflexión especular a aquella reflexión que se produce cuando la superficie de un material es perfectamente lisa y plana (microscópicamente hablando), haciendo que los rayos (o haces) de luz incidentes y reflejados tengan el mismo ángulo respecto de la normal de la superficie de reflexión. 


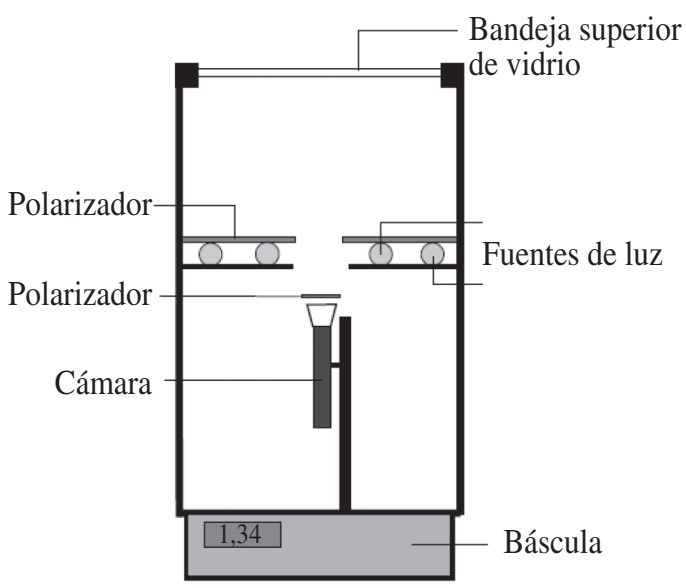

Figura 2. Prototipo desarrollado por IBM [5].

imagen, capturan una fotografía sin iluminación y otra fotografía con la luz fluorescente; así, examinan qué pixeles han aumentado de brillo según un valor de umbral, clasificando entonces los pixeles que se encuentren por encima de este umbral como pixeles del producto y por debajo como pixeles del fondo.

El espacio de color utilizado para extraer esta característica de la fruta o verdura es el mismo espacio utilizado en el artículo [7], HSI, y como herramienta de representación utilizan los histogramas, obteniendo entonces un histograma unidimensional para cada componente del espacio HSI por separado y al final se crea un histograma extendido unidimensional, que concatena los histogramas de cada componente de color individuales.

Además de este proceso, extienden el concepto de histogramas de color a histogramas de textura y otras características. La técnica de clasificación utilizada es la de vecinos más cercanos buscando desarrollar un sistema de fácil entrenamiento (como es requerido en un supermercado).

El sistema presenta, según los autores, una exactitud del $95 \%$ en el reconocimiento del producto correcto (este análisis lo realizan en un escenario de 4 posibles opciones).

En 1996, cuando fue desarrollado este sistema, las cámaras y los procesadores digitales de señales tenían un costo elevado, por tal motivo la implementación masiva del sistema en las cajas registradoras de los supermercados no fue exitosa [8].
En países como Malasia donde se ha pensado revitalizar el sector agrícola para convertirlo en parte del motor de crecimiento económico [9] se está viendo que las tecnologías de la información y comunicación (TIC) son una gran herramienta para mejorar la situación del sector agrícola del país, porque la idea de integrar el sector agrícola con tecnologías ha conducido a constantes investigaciones alrededor de la clasificación automática de frutas y verduras, como lo es la investigación realizada por Woo Chaw Seng, Seyed Hadi Mirisaee (2009), los que proponen un nuevo método para el reconocimiento de frutas, indicando que para el proceso de clasificación existen diferentes técnicas basadas únicamente en atributos de color y forma; sin embargo, plantean que diferentes frutas pueden presentar valores similares de color y forma haciendo que aún no se tengan métodos robustos y efectivos para identificar y distinguir imágenes de frutas [4].

Plantean entonces un nuevo método, el que analiza tres características: color, forma y tamaño, con el fin de incrementar la exactitud y la repetibilidad del reconocimiento. Con el fin de obtener mejores resultados se trabaja con el espacio de color RGB y se utilizan como algoritmo de clasificación los vecinos más cercanos KNN. El método propuesto alcanza un éxito del $90 \%$.

Otra propuesta interesante es la desarrollada por Anderson Rocha, Daniel C. Hauagge, Jacques Wainer y Siome Goldenstein (2010) en [2]; documento que empieza señalando el problema del reconocimiento de patrones de manera general, describiendo que muchos de estos problemas son difíciles de solucionar utilizando un solo descriptor de características, por tal razón, en muchas ocasiones se hace obligatoria la fusión de varios descriptores para poder llegar a un reconocimiento satisfactorio.

En este punto se propone entonces un nuevo enfoque, porque a pesar de que la fusión de características puede funcionar bien para ciertas aplicaciones, en este caso puede producir resultados inesperados cuando las frutas no son debidamente normalizadas y preprocesadas, adicionando también que se pueden necesitar más datos de entrenamiento.

Para dar solución a este requerimiento se propone un enfoque unificado que combina varias características y clasificadores, requiriendo menos datos de 
entrenamiento, siendo más adecuado que un método sencillo, donde todas las características son concatenadas e introducidas de forma independiente para cada algoritmo de clasificación [2]. La comprobación de este enfoque se realiza sobre un problema multiclase como lo es el reconocimiento de frutas, donde no se presenta una iluminación uniforme, utilizando descriptores estadísticos de color y textura así como un enfoque estructural con descriptores de apariencia. Todos los enfoques discutidos en este documento se implementaron en el espacio de color HSV; por último se indica que este enfoque requiere más capacidad de almacenamiento.

\section{Sistema de luz controlada y posicionamiento de cámara}

Dentro de todo sistema de visión artificial la iluminación juega un papel fundamental, ya que al tener unas condiciones de luz adecuadas se puede mejorar la exactitud, la fiabilidad y los tiempos de respuesta del sistema, al poder ejecutar algoritmos más livianos.

Este sistema tiene como objetivo no depender de la luz ambiente, pues es una variable difícil de controlar al trabajar en distintos recintos con iluminaciones diferentes que puedan alterar las características de las frutas a evaluar, o producir sombras que afecten el algoritmo de segmentación y se obtengan formas diferentes a la forma de la fruta real.

Para solucionar estos inconvenientes y conseguir un sistema de luz que genere una iluminación uniforme en las frutas se ha implementado un sistema de luz en anillo que contiene la cámara en su centro, de este modo se logra evitar la presencia de sombras en la imagen procurando que el algoritmo de segmentación sea de bajo costo computacional y de rápida respuesta, tal como se muestra en la Figura 3.

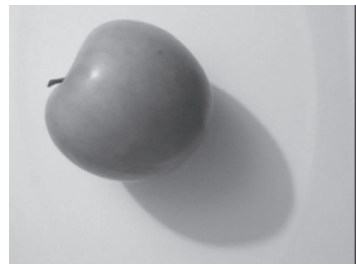

a) Sombra en imagen $\sin$ sistema de iluminación en anillo

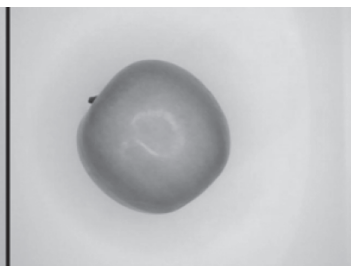

b) Anulación de sombra con sistema de iluminación en anillo
Figura 3. Toma de imagen con y sin sistema de iluminación.
En la Figura 4 se puede observar el diseño de la plataforma final compuesta por el sistema de medida de masa, el sistema de luz en anillo y la cámara posicionada en el centro del anillo del sistema de iluminación, permitiendo entonces realizar el procesamiento de las imágenes de las frutas con condiciones controladas.

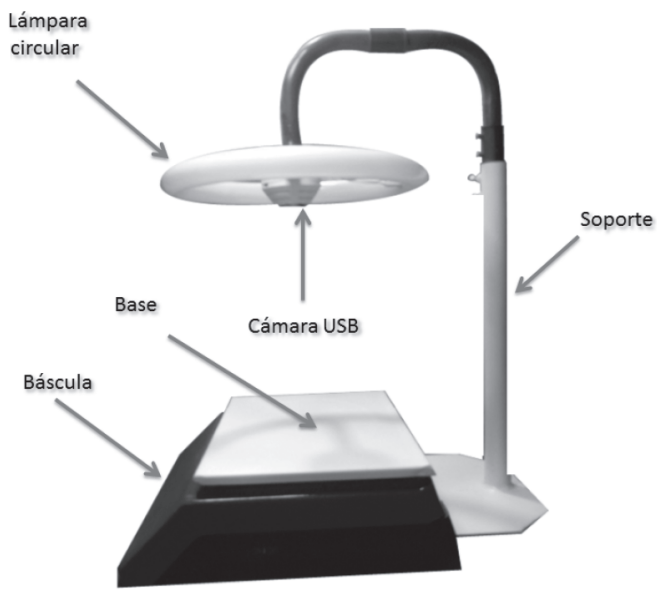

Figura 4. Plataforma física del sistema de visión construido.

\section{Construcción de la base de datos}

Como el objetivo del sistema es identificar frutas que se encuentran comúnmente en un supermercado se tomó una muestra de la población total de frutas típicas colombianas, esta está compuesta por 9 clases que servirán de base para validar la hipótesis, en la que se expresa "que es posible dotar a una báscula electrónica con la capacidad de realizar la percepción autónoma e identificar diferentes clases de frutas".

Las 9 clases de frutas son: manzana verde, manzana roja, mango Tommy, naranja Tangelo, maracuyá, granadilla, kiwi, guayaba y banano.

Para construir la base de datos necesaria para el entrenamiento del algoritmo de clasificación se tomaron muestras del tipo de frutas antes mencionadas de varios supermercados de la región.

La adquisición de las imágenes se realizó con una cámara web de Creative Labs sobre la plataforma de la Figura 4 con una resolución de 640x480, y se almacenaron en el espacio de color RGB. 
La base de datos se compone en su totalidad por 90 imágenes, donde cada clase de frutas hace presencia con 10 muestras.

\section{Visión por computador-tratamiento de las imágenes de frutas}

Para detectar la fruta presente en la escena se utilizó la técnica de segmentación mediante umbral sobre la capa de color azul del modelo RGB aprovechando la circunstancia de tener un fondo blanco.

Como se observa en la Figura 5, la parte de la fruta en la capa azul genera un alto contraste contra el fondo haciendo posible obtener una buena segmentación de la fruta utilizando un valor de umbral bimodal, ello se manifiesta observando el histograma de la imagen, en la que se aprecian dos elevaciones considerables, acompañadas de un valle, que representan el objeto y el fondo.
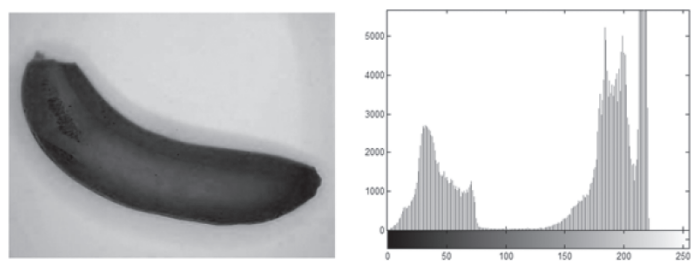

Figura 5. Capa azul de la imagen de un banano. Análisis realizado en el modelo RGB.

Por lo tanto el procedimiento para lograr la segmentación comienza extrayendo la capa azul del modelo RGB de la imagen original, luego sobre esta imagen se calcula el valor de umbral utilizando el algoritmo global de OTSU mediante la función graythresh de Matlab sobre la capa azul de la imagen RGB de la fruta seleccionada [10-11].

Luego de tener el valor umbral se procede a la binarización global de la capa azul con la función im2bw de Matlab, la que transforma la imagen de capa azul del modelo RGB en una nueva imagen binaria ( 1 y 0 ) con base en el umbral calculado anteriormente. El resultado obtenido se puede observar en la Figura 6.

Esta nueva imagen contiene únicamente unos y ceros y representa muy bien la forma y posición de la fruta en la escena; siendo esta la base para obtener la máscara que permitirá identificar y eliminar los pixeles del fondo que presentan información que no es de interés. Como la imagen está compuesta

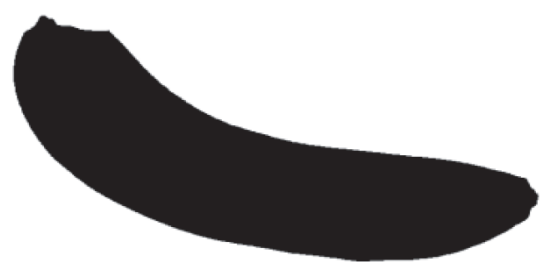

Figura 6. Imagen binaria de la capa azul del modelo RGB. Análisis realizado para un banano mediante el algoritmo global de OTSU.

de ceros y unos, siendo el negro representado por ceros y el blanco por unos, se puede multiplicar esta imagen segmentada por cada capa de color R, G y B que compone la imagen original de la fruta a color, con esto se está eliminando la información de los pixeles que componen la fruta porque estos serían multiplicados por cero, y en cambio se estarían salvando los pixeles del fondo. Para corregir esta situación se invierten los valores de los bits de la imagen binaria y así se obtiene la nueva máscara, como se ilustra en la Figura 7.

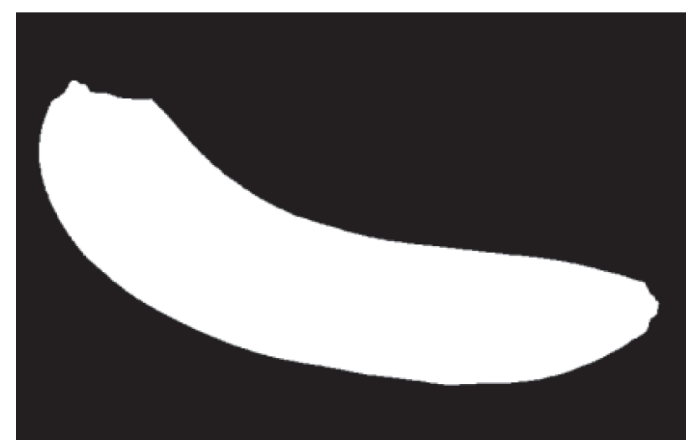

Figura 7. Imagen de la Figura 6 con los bits invertidos.

Con la máscara, mostrada en la Figura 7, es posible eliminar los pixeles del fondo y obtener los pixeles que componen la fruta, realizando básicamente una multiplicación entre ella y cada una de las capas y luego concatenándolas nuevamente para formar la imagen a color, con lo que se obtiene la imagen de la fruta segmentada como se observa en la Figura 8.

La Figura 8 presenta una buena segmentación, ya que la superficie del banano no tiene muchas reflexiones especulares gracias a su textura, pero en frutas como el maracuyá donde su superficie es más lisa, se pueden dar reflexiones importantes que deben ser corregidas, pues hacen que en el proceso 

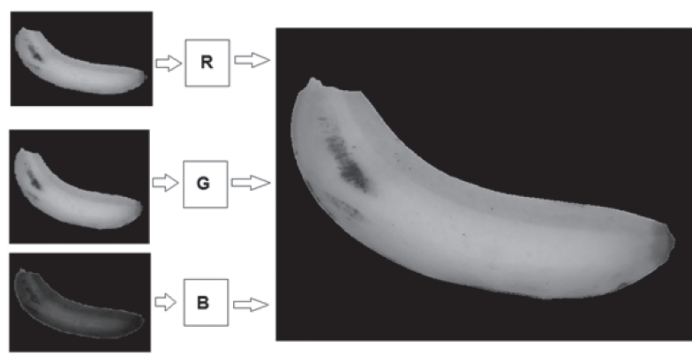

Figura 8. Concatenación y formación de la imagen de la fruta. Imagen segmentada en el modelo RGB.
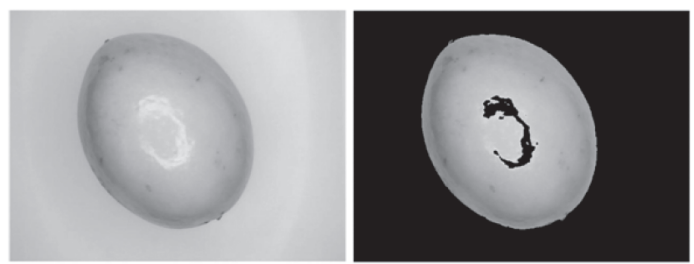

Figura 9. Reflexiones especulares en un maracuyá. Las reflexiones representan depresiones en la segmentación.

de segmentación resulten concavidades no deseadas en la fruta, como se aprecia en la Figura 9.

La técnica revisada en la literatura para corregir este tipo de imperfecciones es la realización de un procesamiento morfológico.

Para esto se creó un elemento estructurante SE tipo disco, ya que con este se obtuvieron bordes más suaves que se ajustan a la forma curva regular de las frutas comparados con los otros elementos probados como el tipo cuadrado que dejaba bordes con cambios más bruscos.

Experimentalmente se encontró que el tamaño del radio debe ser de magnitud 11 para eliminar por completo las imperfecciones en el interior de la fruta.

En la Figura 10 se ilustra el elemento estructurante tipo disk de tamaño 11 utilizado.

La función morfológica que opera con el elemento estructurante y elimina las imperfecciones de las máscaras se conoce como operador de cierre (closing).

Los investigadores usaron la función de Matlab Imclose sobre la imagen binarizada a usar como

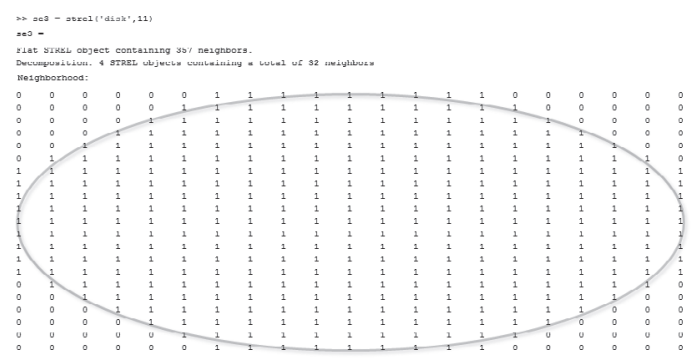

Figura 10.Elemento estructurante tipo disco de tamaño 11

máscara mediante el elemento estructurante $\mathbf{S E}$ definido anteriormente.

La nueva imagen sin las concavidades, creadas por las reflexiones especulares que fueron de difícil eliminación en la binarización de la capa azul del modelo RGB del maracuyá de la Figura 9, se aprecia en la Figura 11.

La Figura 11 se encuentra correctamente segmentada, pues se han eliminado los agujeros provocados por las reflexiones especulares, pero sigue presentando en el borde un resplandor de color blanco, el que para el procesamiento posterior que consiste en la extracción de los diferentes descriptores que caracterizan cada fruta como el color, puede afectar las medidas; por este motivo se reduce el tamaño de la fruta segmentada para eliminar este borde conservando su forma original.

Para lograrlo se utiliza otro operador morfológico conocido como erosión, este se realiza con la

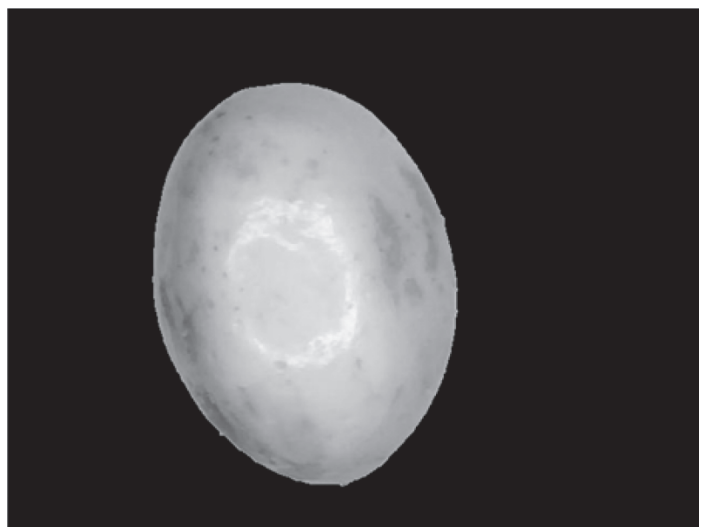

Figura 11. Imagen con corrección de reflexiones especulares. En este caso se utiliza procesamiento morfológico closing. 
instrucción imerode de Matlab sobre la última imagen máscara cerrada (closing).

El elemento nuevo estructurante usado para la erosión es también de tipo disco, pero de radio 7. Este resultado se comprobó de forma experimental, verificándose que era el tamaño adecuado para eliminar el borde blanco.

El resultado final se observa en la Figura 12.

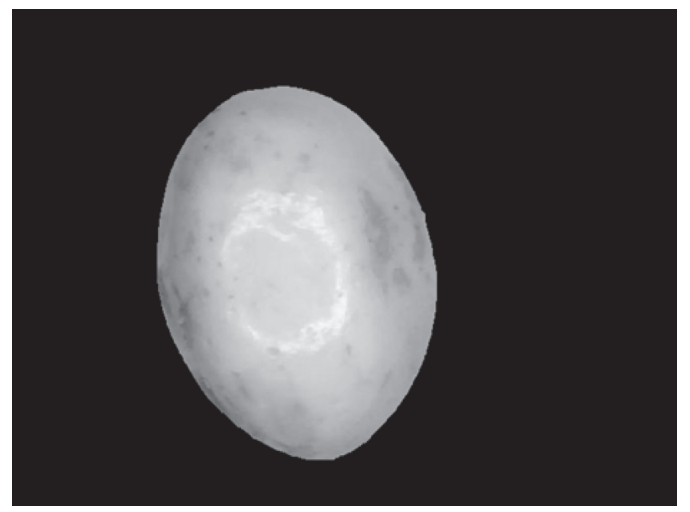

Figura 12. Imagen con borde blanco anulado. En este caso se utiliza el operador morfológico de erosión con elemento estructurante tipo disco de radio 11 .

\section{Descriptores}

Para el proceso de descripción, el que consiste en obtener los patrones determinantes que permitieran diferenciar un tipo de fruta (clase) de otro distinto, se tuvieron en cuenta el color, la forma y el tamaño.

Las distintas características utilizadas para medir estas variables fueron: el área, el perímetro, la redondez, el eje mayor, el eje menor y el valor medio de los canales HSV y RGB.

Los valores de los descriptores se realizaron utilizando la función regionprops de Matlab, esta mide un conjunto de propiedades dentro de las diferentes regiones identificadas como objetos dentro de una imagen binaria. Para indicarle a la función qué regiones se encuentran en la imagen se utiliza la función bwlabel de Matlab, la que retorna una matriz etiquetada $L$ del mismo tamaño de la imagen, con distintas etiquetas numeradas que marcan la existencia de los diferentes objetos presentes en la imagen, e indican a la función regionprops sobre qué regiones de las etiquetadas debe realizar el cálculo de las características a usar como descriptores.

En la solución implementada, al aplicar la función bwlabel se trabajó con conectividad 8 , y se generó la matriz $L$ de referencia para la función regionprops y se creó una variable de nombre descriptor, esta almacena el resultado de todas las características entregadas por regionprops. Las características necesarias para formar el patrón característico de cada fruta se recuperaron y se almacenaron en las variables area, perimetro, redondez, ejemayor, ejemenor y valores medios de los canales HSV y RGB (nótese que no llevan tilde), de la siguiente manera:

- $\quad$ area=descriptor.Area

- perimetro=descriptor.Perimeter

- $\quad$ redondez $=4 * \mathrm{pi}^{*}($ area/perimetro^ 2$)$

- ejemayor=descriptor.MajorAxisLength

- ejemenor=descriptor.MinorAxisLenght

Además se extrajeron la media de los canales de los modelos de color HSV y RGB.

\section{Reconocimiento de objetos}

En busca de acercar a las máquinas a la capacidad de aproximación y decisión que poseen los seres humanos en tareas de reconocimiento, se ha desarrollado una amplia teoría alrededor de los sistemas o modelos encargados de esta función, conocidos como clasificadores.

En este apartado se trabajaron los clasificadores KNN y bayesiano, donde para cada uno de ellos se presenta una breve descripción.

\section{Clasificadores usados}

Clasificador K-NN. Este clasificador es una extensión de la regla de clasificación NN, esta es conocida como vecino más cercano (Nearest Neighbour). La idea fundamental en la que se basa la regla del vecino más cercano reside en considerar que las muestras pertenecientes a una misma clase se encuentran probablemente próximas en el espacio de representación. Para determinar la cercanía entre muestras, la regla utiliza el concepto métrica de distancia, donde existen diferentes maneras de realizar esta medición. Por lo tanto, debido al conjunto de $\mathrm{N}$ muestras pasadas con clases definidas y a causa de una muestra nueva $x$ de la que se desconoce su clase, 
esta se clasificará o etiquetará con la clase en la que la medida de la distancia de la nueva muestra $x$, con un elemento de la clase seleccionada, presentó el valor mínimo. Este procedimiento no aprovecha toda la información que se puede extraer del conjunto de entrenamiento, por tal motivo se crea la regla K-NN, la que además de encontrar el vecino más cercano a la nueva muestra, tiene en cuenta el entorno que rodea a la misma contando el número de muestras $(\mathrm{K})$ que se encuentran próximas. Así, si se tiene un conjunto de muestras para cada clase, la clasificación se basa en las $\mathrm{K}$ muestras más próximas a la nueva muestra.

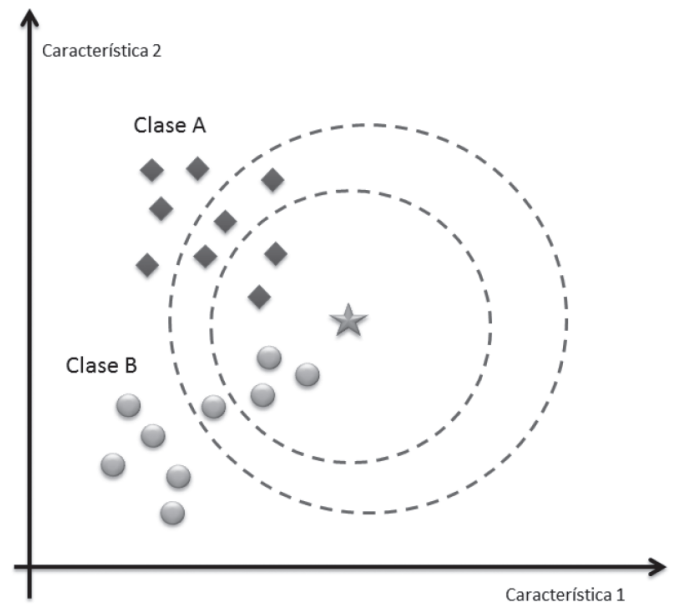

Figura 13. Ejemplo clasificación K-NN.

Observando la Figura 13, la primera vecindad encerrada con el círculo más pequeño $(\mathrm{k}=5)$ indica que la nueva muestra (forma de estrella) será clasificado como círculo, debido a que en esta región hay tres círculos contra dos rombos, pero si se selecciona una región mayor como el círculo punteado más alejada de la estrella $(\mathrm{k}=9)$, la estrella será clasificada como rombo, pues en esta región hay cinco rombos contra cuatro círculos [12].

Clasificador bayesiano. Esta clase de algoritmo pertenece a la clase de clasificadores supervisados; es un clasificador probabilístico basado en el teorema de Bayes, siendo un método importante no solo porque ofrece un análisis cualitativo de las características y valores que intervienen en el problema, sino porque tiene en cuenta además la importancia cuantitativa de esas características.

Por lo tanto, para este clasificador un objeto con ciertas características distintivas hará parte de una determinada clase si la probabilidad de pertenecer a esta clase es mayor a la probabilidad de pertenecer a cualquier otra clase [6], como se ilustra en la ecuación (1).

$X \in \Psi_{i} s i$

$$
P\left(\omega_{i}\right) p\left(X \mid \omega_{i}\right)>P\left(\omega_{j}\right) p\left(X \mid \omega_{j}\right)
$$

Donde $\Psi$ es el espacio de características que está dividido en regiones $\Psi_{i}, i=1,2, \ldots, \mathrm{N}$, donde $\mathrm{N}$ es el número de clases. $P\left(\omega_{i}\right)$ es la probabilidad $a$ priori, por la que un objeto con características $X$ pertenece a la clase $\omega_{i}$ y $p\left(X \mid \omega_{i}\right)$ es la función de probabilidad condicional de la clase $\omega_{i}$ para $X$.

En la práctica, las funciones de probabilidad no se conocen y por lo tanto se deben estimar, para ello primero se asume la forma de la función de probabilidad, y luego se hallan sus parámetros a partir del conjunto de entrenamiento. Se supone que las funciones de probabilidad de las clases están descritas por distribuciones normales o gausianas [6].

Luego de haber extraído las características determinantes que permiten describir las diferentes frutas y haber definido las técnicas a usar, se puede realizar la clasificación.

Para ello es necesario entrenar el clasificador, para realizarlo se hizo uso de la base de datos construida con las diferentes imágenes de frutas y se realizó la segmentación y extracción de las características, para que, de esta forma, el clasificador vaya aprendiendo qué valores son los representativos para cada fruta en especial.

Para validar el clasificador se utilizó el método de validación cruzada, conocido como k-fold, donde los datos de la muestra se dividen en K subconjuntos, utilizando k-1 conjuntos para el entrenamiento y un solo subconjunto para prueba.

El valor de $\mathrm{K}$ es 10 , pues se observó que es el valor que comúnmente se utiliza para validar los datos de entrenamiento.

Se implementaron los clasificadores supervisados KNN (K Nearest Neighbors) y el bayesiano, por resultar altamente recurrentes como clasificadores adecuados y su simplicidad de implementación en Matlab. 


\section{Entrenamiento}

La matriz de entrenamiento definida es común para ambos clasificadores:

- caracte es la matriz que contiene las características de cada fruta. Si estas son representadas con las características anteriores, suponiendo que se tienen $\mathrm{N}$ imágenes de entrenamiento, esta matriz es de tamaño $\mathrm{Nx} 9$.

- clases es una matriz de tamaño Nx1 que indica a qué clase pertenece cada nueva fruta; por ejemplo, si $\mathrm{N}=90$ y hay 10 guayabas, 10 bananos, 10 naranjas, 10 mangos, 10 manzanas rojas, 10 manzanas verdes, 10 granadillas, 10 kiwis y 10 maracuyás, la matriz se representa de la siguiente manera [1 11111111111122 $222222223333333333 \ldots 99999$ $99999]^{\mathrm{t}}$. El 1 representaría las guayabas, el 2 los bananos y así respectivamente.

\section{Clasificación}

En el proceso de clasificación, la función del algoritmo es entregar el número que representa la clase a la que pertenece la fruta que se está analizando; por ejemplo, si se ingresa una nueva imagen al sistema y se realiza el proceso de segmentación, extracción de características y clasificación y el resultado es 2, quiere decir, siguiendo el ejemplo anterior, que la fruta presente en la imagen pertenece a la clase del banano.

La muestra es una matriz de $1 \mathrm{xN}$ que contiene las $\mathrm{N}$ características que describen la fruta que se desea identificar. Lo que hace la función de clasificación es buscar, después de que ha sido entrenado, la clase a la que pertenece la nueva fruta en análisis.

Una vez obtenido el resultado se muestra en pantalla el tipo de fruta y su masa respectiva.

\section{RESULTADOS}

En este apartado se dan a conocer los resultados obtenidos tanto en el diseño de la plataforma como en la clasificación.

\section{Plataforma final}

La plataforma finalmente construida brindó la posibilidad de tener un espacio de trabajo con condiciones controladas desde distancia al objeto y de luz, pues se logró tener una iluminación uniforme en las frutas y la no presencia de sombras considerables que afectaran el proceso de segmentación de la imagen, ello permitió la implementación de un algoritmo de segmentación rápido que analiza los objetos en la imagen considerando únicamente su nivel de gris.

\section{Reconocimiento de frutas}

Para medir el rendimiento de los clasificadores se dividieron las imágenes en un conjunto de entrenamiento y otro de prueba, estos se encontraban conformados por 80 y 10, respectivamente.

En las gráficas de las Figuras 14, 15, 16 y 17 se presentan los porcentajes de acierto sobre las imágenes del conjunto de prueba.

\section{Clasificador KNN}

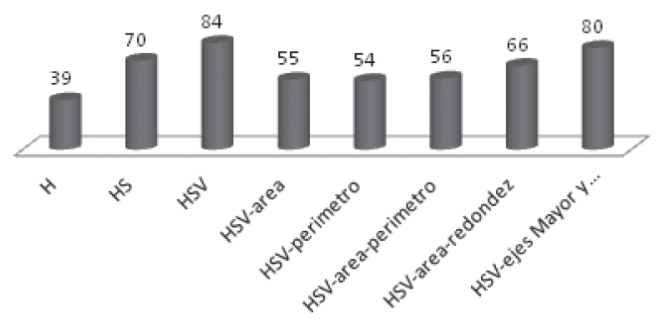

Figura 14. Porcentaje de acierto del clasificador KNN. Análisis para distintas combinaciones de características, especialmente utilizando el espacio de color HSV.

\section{Bayes}

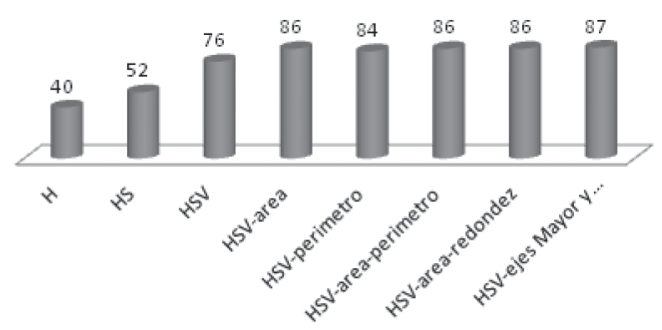

Figura 15. Porcentaje de acierto del clasificador bayesiano. Análisis para distintas combinaciones de características, especialmente utilizando el espacio de color HSV. 


\section{KNN RGB}

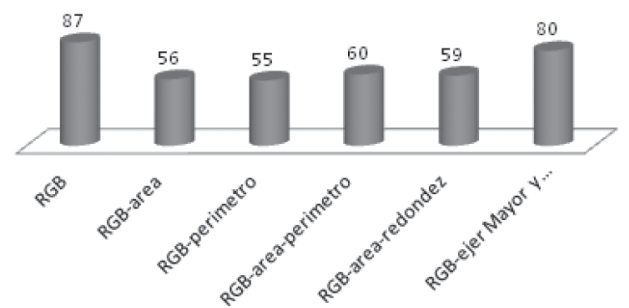

Figura 16. Porcentaje de acierto del clasificador KNN. Análisis para distintas combinaciones de características, especialmente utilizando el espacio de color RGB.

\section{Bayes RGB}

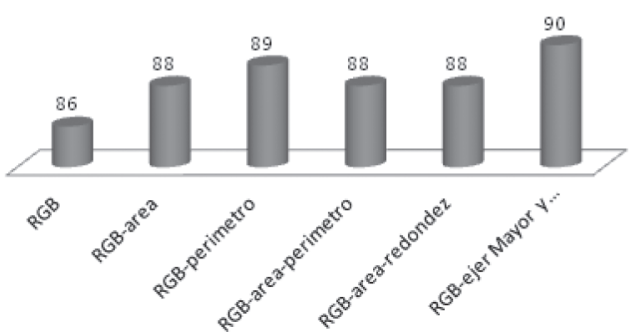

Figura 17. Porcentaje de acierto del clasificador bayesiano. Análisis para distintas combinaciones de características, especialmente utilizando el espacio de color RGB.

Tomando como base la literatura estudiada se observó el uso frecuente de clasificadores supervisados específicamente con las técnicas KNN (K Nearest Neighbors) y bayesiano [4-6], para ello se experimentó con distintas combinaciones de las características extraídas que describen cada tipo de fruta.

En las Figuras 14, 15, 16 y 17 es posible observar que no siempre por usar todos los parámetros se obtienen mejores resultados; por ejemplo, al analizar los resultados obtenidos con el clasificador KNN en la Figura 14, se aprecia que se presenta una tasa de acierto mucho mayor utilizando únicamente las características de color HSV comparada con la adición del área y perímetro que hacen que la tasa disminuya a $56 \%$, dato que se esperaría que mejorara debido a que hay más información de descripción, pero sucede lo contrario; esto demuestra que los clasificadores presentan un alto porcentaje de acierto utilizando determinados parámetros debido a la forma en que opera su algoritmo.

En general se observa que el clasificador bayesiano tiene una mejor eficacia frente al clasificador KNN, y es con este que se obtiene el mayor porcentaje de acierto con un valor del $90 \%$ utilizando los valores medios de las intensidades del espacio de color RGB y los valores de los ejes mayor y menor (véase la Figura 17). También es importante resaltar comparando las Figuras 14, 15, 16, y 17, que trabajando en el espacio de color RGB se obtuvieron mejores resultados que trabajando en el modelo HSV. En la Figura 18 se presenta una imagen del aplicativo que realiza la clasificación de las frutas en funcionamiento.

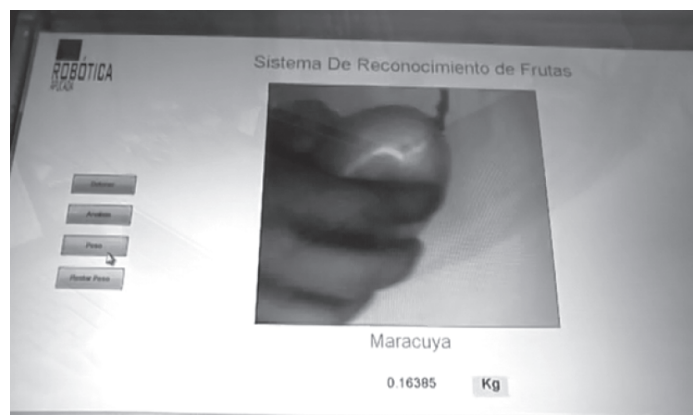

Figura 18. Pantalla capturada del clasificador de frutas. Presentación de la operación del sistema.

\section{Propuestas para proyectos futuros}

Para trabajos futuros que deseen lograr mejores resultados y ampliar los alcances de este desarrollo se expondrán a continuación algunas posibles mejoras que se vislumbraron durante la terminación del proceso investigativo.

Sería interesante usar una cámara inalámbrica, que permitiera el procesamiento de la imagen en un computador remoto, brindando la posible implementación de este tipo de básculas en supermercados, donde se dispongan varios de estos dispositivos y el procesamiento de sus datos se realice en un computador central que esté en un lugar distinto al lugar donde se encuentran las básculas.

Para probar el sistema se utilizaron 9 clases de frutas; un trabajo futuro podría tratar de incrementar este número de clases y para lograr discernir entre frutas que tienen un alto grado de similitud se podrían 
implementar técnicas de descripción más robustas que brinden mayor información con características más discriminativas.

Una propuesta diferente al tener un computador remoto que realice el procesamiento de los datos entregados por la báscula sería implementar un sistema de procesamiento de imagen dentro de la misma, teniendo procesamiento local, este podría ser un sistema de altas prestaciones como un DSP o una FPGA.

Hasta ahora solo se reconoce una fruta de manera individual, pero generalmente los consumidores de un supermercado seleccionan mayores cantidades, por este motivo, un trabajo futuro sería reconocer y segmentar las frutas presentadas en distintas cantidades.

\section{CONCLUSIONES}

Se ha desarrollado un sistema electrónico con capacidad de reconocer diferentes clases de frutas mediante visión por computador.

Como se observó, la probabilidad de acierto en el mejor de los casos es bastante elevada, alrededor de 90\%; haciéndolo robusto al reconocer las 9 clases de frutas utilizadas.

La plataforma construida además de tener un enfoque a la clasificación de frutas, está orientada a desarrollar un proceso de investigación en el que se puedan posicionar objetos diferentes y estudiar técnicas de visión artificial y algoritmos de clasificación, pues brinda un ambiente con condiciones controladas.

Una de las contribuciones de este trabajo es la creación de una importante base de datos de frutas sobre las cuales se puedan aplicar y estudiar técnicas de visión artificial más sofisticadas o sirva como herramienta pedagógica en cátedras de procesamiento de imágenes donde los estudiantes puedan probar sus algoritmos.

Después de revisar la literatura que trataba sobre esta problemática se encontró que el modelo de color más utilizado para interpretar o describir los colores en una escena es el modelo HSI, por este motivo se esperaba que los mejores resultados se presentaran utilizando este modelo; sin embargo el mayor porcentaje de acierto se presentó utilizando el modelo RGB. Este resultado es importante debido a que colocó en mejor posición al modelo RGB respecto al HSI al momento de describir las nueve clases de frutas utilizadas.

Se comprobó que no siempre al seleccionar la mayor cantidad de características los clasificadores entregan la mejor respuesta, pues es importante revisar que las características que describen los objetos a clasificar presenten un valor bajo de correlación, indicando que contienen la mayor información que discrimina a cada clase en particular; por lo tanto para esta clase de frutas las características que mejor las describieron fueron los valores medios de los canales RGB y la longitud del eje mayor y menor.

\section{REFERENCIAS}

[1] R. Pathak and S. Joshi. "Recent Trends in RFID and a Java based Software Framework for its Integration in Mobile Phones". First Asian Himalayas International Conference on Internet AH-IC2009 Hyatt Regency Kathmandu, Kathmundu, Nepal. November 3-5, 2009.

[2] A. Rocha, D. C. Hauagge, J. Wainer and S. Goldenstein. "Automatic fruit and vegetable classification from images". ScienceDirect Computers and Electronics in Agriculture, pp. 96-104. 2010.

[3] G. Roussos. "Enabling RFID in retail". Computer. Vol. 39, Issue 3, pp. 25-30. March, 2006. ISSN: 0018-9162.

[4] W. Chaw Seng and Seyed Hadi Mirisaee. "A New Method for Fruits Recognition System". IEEE Conference Publication International Conference on Electrical Engineering and Informatics, pp. 130-134. 2009.

[5] R.M Bolle, J.H Connell, N.Haas, R. Mohan and G.Taubin. "VeggieVision: A Produce Recognition System". IEEE Conference Publications, pp. 244-251. 1996.

[6] Z. Sandoval and F. Prieto. "Procesamiento de imágenes para la clasificación de café cereza”. Prospectiva Vol. $7 \mathrm{~N}^{\circ}$ 1, pp. 67-73. Enero-Junio de 2009.

[7] G. Kay and G. de Jager. "A versatile colour system capable of fruit sorting and accurate object classification". IEEE Conference Publications, pp. 145-148. 1992. 
[8] R.M. Bolle, J. Connell, N. Haas, R. Mohan and G. Taubin. "Veggie Vision". Date of visit: November 4, 2012. URL: http://researcher. ibm.com/view_project.php?id= 2021

[9] N.B. Ahmad Mustafa, S. Khaleel Ahmed, Z. Ali, W. Bing Yit, A.A. Zainul Abidin and Z.A. Md Sharrif. "Agricultural Produce Sorting and Grading using Support Vector Machines and Fuzzy Logic". IEEE Conference Publications, pp. 391-396. 2009.
[10] R. González and R. Woods. "Digital Image Processing". Prentice-Hall. 3rd edition. 2007.

[11] MathWorks. "Image Processing Toolbox User's Guide". Date of visit: April 16, 2014. URL: http://www. mathworks.com/products/ image/description 1.html

[12] C.M. Bishop. "Pattern recognition and machine learning". Springer. Vol. 1. New York, USA. 2006. 